Vessel failures predominantly manifested as the need for repeat revascularization; $5.6 \%$ of patients implanted with a sirolimus-eluting stent underwent subsequent percutaneous coronary intervention or bypass grafting, compared with $13.4 \%$ from the bare-metal stent group $(P<0.001)$. There were no significant differences in the rates of death, recurrent $\mathrm{MI}$ or stent thrombosis between the groups.

A subset of 174 patients was followed up with an angiogram 8 months after implantation of the original stent. Sirolimus-eluting stents lost significantly less of their lumen diameter $(0.14 \pm 0.49 \mathrm{~mm}$ vs $0.83 \pm 0.52 \mathrm{~mm} ; P<0.001)$ and were less likely to be affected by restenosis (3.5\% vs $20.3 \%, P=0.001)$. The authors suggest that the benefit conferred by these drugeluting stents might, therefore, be a function of suppression of neointimal proliferation.

In the second trial-PASSION-Laarman and colleagues compared patients fitted with paclitaxel-eluting (TAXUS ${ }^{\circledR}$ Express $^{2 \circledR}$; Boston Scientific) versus uncoated (TAXUS ${ }^{\circledR}$ Express $^{2 \circledR}$ or Liberté ${ }^{\circledR}$; Boston Scientific [Maple Grove, MN]) stents. The researchers enrolled 619 patients from 2 centers in The Netherlands between March 2003 and December 2004.

After 1 year, there were no statistically significant differences between the two groups with regard to rates of revascularization or CABG of the implanted vessel, in-stent thrombosis, recurrent $\mathrm{Ml}$, or death. There was, however, a trend towards better outcomes in the paclitaxel-eluting stent group; for example, major adverse cardiac events affected $8.8 \%$ of patients with these stents, compared with $12.8 \%$ of those who received conventional devices.

The results of the PASSION trial are in contrast to those of previous studies, which have shown paclitaxel-eluting stents to be significantly superior to uncoated stents, at least for elective percutaneous coronary intervention. The PASSION trial, however, included patients with larger arteries and fewer patients with diabetes. Subjects in this trial could, therefore, have been less likely to experience restenosis than those in other trials. Differences in study design and patient characteristics, as well as variance in inclusion and exclusion criteria, make direct comparison of the TYPHOON and PASSION trials problematic.

Original articles Spaulding C et al. (2006) Sirolimus-eluting versus uncoated stents in acute myocardial infarction. N Engl JMed 355: 1093-1104

Laarman GJ et al. (2006) Paclitaxel-eluting versus uncoated stents in primary percutaneous coronary intervention.

N Engl J Med 355: 1105-1113

\section{Sphygmomanometer calibration could increase the accuracy of hypertension detection}

Poorly calibrated sphygmomanometers could lead to clinically significant errors in hypertension detection, over and above errors introduced as a result of random intra-individual blood pressure (BP) variability. To determine the proportion of error attributable to inadequate sphygmomanometer calibration, Turner et al. carried out a computer simulation study.

The investigators modeled BP measurements in a population of 15 million adult Australians using 17,000 calibrated and 17,000 uncalibrated sphygmomanometers. The simulation showed that, as the number of visits to the clinician increased, the proportion of hypertensiondetection error attributable to uncalibrated sphygmomanometers also increased; the effect of this source of error was partially obscured by random BP variability when the number of consultations in an individual was small. After three patient visits, poorly calibrated sphygmomanometers accounted for $20 \%$ of undetected systolic and $28 \%$ of undetected diastolic hypertension (BP >140/90 mmHg) and for $15 \%$ of falsely detected systolic and $31 \%$ of falsely detected diastolic hypertension. The model indicated that error attributable to poor sphygmomanometer calibration is more likely to compromise detection of hypertension in young adults (18-24 years old) and women.

More frequent and rigorous calibration of sphygmomanometers by accredited organizations would be a cost-effective way of improving the accuracy of hypertension detection.

Original article Turner MJ et al. (2006) Lack of sphygmomanometer calibration causes over- and underdetection of hypertension: a computer simulation study. J Hypertens 24: 1931-1938 